Article

\title{
Time Coding OTDM MIMO System Based on Singular Value Decomposition for 5G Applications
}

\author{
Rana M. Aly, Amira Zaki $@$, Waleed K. Badawi and Moustafa H. Aly *(i) \\ College of Engineering and Technology, Arab Academy for Science, Technology and Maritime Transport, \\ Alexandria 1029, Egypt \\ * Correspondence: mosaly@aast.edu; Tel.: +20-100-663-9473
}

Received: 14 May 2019; Accepted: 17 June 2019; Published: 2 July 2019

\begin{abstract}
For 5G and beyond cellular communication systems, new coding and modulation techniques are suggested to reach the requirements of high data rate and quality of service. In this paper, a new space-time coded orthogonal transform division multiplexing (STC OTDM) technique is proposed for 5G applications. The proposed system is used to enhance the data rate and performance of the orthogonal transform division multiplexing (OTDM) technique. The proposed system is based on using space-time coding (STC) with OTDM to increase the system diversity and consequently the system performance. The OTDM technique is based on transmitting data on orthogonal basis functions obtained from the Singular Value Decomposition (SVD) of the channel impulse response of the desired user. Various modulation techniques like QPSK, 64-QAM, and 256-QAM are investigated using different subcarriers and channel models. The simulation results show that the proposed system achieved a better performance when compared to classical and recent multicarrier techniques. The proposed technique increases the diversity gain resulting in a decrease in the fading effect of the multipath channel and an enhancement in the bit error rate (BER) performance. The proposed technique also provides a secure data transmission to the desired user as his data is sent on the basis functions extracted from his own channel impulse response that cannot be decoded by other users.
\end{abstract}

Keywords: bit error rate (BER); space-time coding (STC); Singular Value Decomposition (SVD); massive MIMO; orthogonal transform division multiplexing (OTDM); Toeplitz matrix

\section{Introduction}

Wireless systems suffer from a rapid increasing demand for high data rates and quality of service. Therefore, it is substantial to design a practical physical layer technique to provide high data rates with high system performance [1]. This technique provides reliable advanced communication system like 5G and beyond [2]. This paper focuses on the cellular communication system although the proposed technique can be extended to different advanced wireless communication networks. The development of several modulation multicarrier techniques that are combined with the massive Multiple-Input-Multiple-Output (MIMO) is essential, which is the key to all the advanced cellular wireless systems [3].

Boroujeny and Moradi showed in [4] that, in the past, orthogonal frequency division multiplexing (OFDM) was one of the most popular modulation technique in wired and wireless systems, due to its ability to combat the frequency selectivity of the transmission channels and achieving high rate without intersymbol interference (ISI) $[5,6]$. Now, new techniques are needed to enhance the bit error rate (BER) and increase diversity.

In [7], Bharti and Rawat showed a combination between the OFDM and Alamouti space time block code (STBC) [8] to increase the performance of BER in multipath fading channels of the wireless 
communication systems by using multiple transmit and receive antennas. Additionally, it extended the diversity order as it used $4 \times \mathrm{N}$ Transmitters-Receivers.

Fernando et al. displayed in $[9,10]$ that the self-heterodyne OFDM (self-het. OFDM) technique gives a better performance than the OFDM. It is used with STC in [9] to enhance the performance of MIMO millimeter wave communication.

In [11] Bariah et al. displayed a non-orthogonal multiple access (NOMA) technique due to its ability to improve the overall spectral efficiency of wireless systems. They show that the maximum possible order of diversity is proportional to the order of the user. The error probability expressions obtained are used to formulate an optimization problem that minimizes the overall bit error rate under power and error rate threshold constrains.

Ghaffari et al. presented in [12] a new non-orthogonal multiple access technique called Sparse Code Multiple Access (SCMA). It offers better performance and higher spectral efficiency than other comparable techniques. However, these improvements come at the expense of complex decoders.

The upcoming $5 \mathrm{G}$ systems are required to provide higher performance and quality of services compared to the currently deployed long-term evolution (LTE) systems. These requirements can be achieved by providing a significant increase in the system date rate, power consumption efficiency, spectral efficiency throughput, size of coverage area, number of devices connecting (users), and low latency. To fulfill the requirements of the coming generations, new techniques, like orthogonal time-frequency space (OTFS) modulation, was proposed by Hadani et al. in [13]. Moreover, the BER has been enhanced by the OTDM technique, besides the improvement of the security which was illustrated by Hamamreh and Arslan in [14].

In this paper, an STC OTDM technique is introduced to meet some of the challenges of the upcoming 5G system by increasing the data rate and the throughput of the system. Moreover, the STC OTDM increases the security level of transmission for the wireless system. A STC OTDM technique is proposed to reduce the BER using the STC, which also increases the diversity order. This is carried out by using Singular Value Decomposition (SVD) of the estimated channel, where every two symbols are modulated using orthonormal basis functions of SVD. Then, the modulated symbols are encoded using an Alamouti encoder for a $2 \times 2$ MIMO system and are transmitted over two consecutive symbol durations. This technique improves diversity and reduces the BER as a result of increasing the signal to noise ratio (SNR), in addition to the advantages of the OTDM mentioned in [15]. This technique is promising for the future high performance and secure $5 \mathrm{G}$ systems when compared to the conventional OFDM system. The proposed technique increases the diversity gain resulting in a decrease in the fading effect of the multipath channel, thus enhancing the bit error rate (BER) performance and throughput of the system. The proposed technique also provides a secure data transmission to the desired user as his data is sent on the basis functions extracted from his own channel impulse response that cannot be decoded by other users, thus the desired user can only receive his data correctly.

The remainder of the paper is organized as follows. Section 2 is devoted to a detailed description of the proposed system. System evaluation and simulation results are presented in Section 3. This is followed by the main conclusions showing the merits of the proposed system in Section 4.

\section{System Model and Analysis}

\subsection{STC OTDM Transmitter}

The proposed system transmitter is illustrated in Figure 1, which shows the sequence of the system transmitter architecture. At the transmitter side, the number of symbols per frame is $\mathrm{N}$. The data symbols $\mathrm{S}$ are transmitted in a number of frames, each of length $\mathrm{N}$ symbols and each frame is divided into two sequences; even sequence, $S_{1}$, and odd sequence $S_{2}$. Both $S_{1}$ and $S_{2} \in \mathrm{C}\left[\frac{\mathrm{N}}{2} \times 1\right]$.

Thus,

$$
\begin{aligned}
& S_{1}=[\mathrm{s}(2(m-1))]^{T} \text { and } \\
& S_{2}=[\mathrm{s}(1+2(m-1))]^{T}
\end{aligned}
$$


where $m=1,2, \ldots, \frac{\mathrm{N}}{2}$

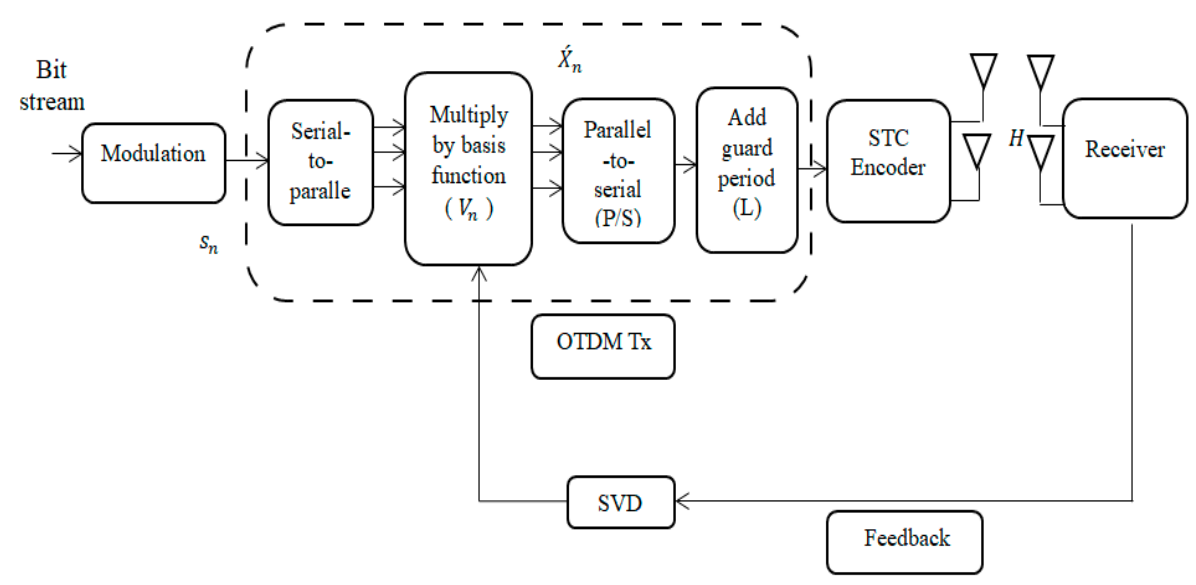

Figure 1. Space-time coded orthogonal transform division multiplexing (STC OTDM) transmitter technique.

After modulation with M subcarriers and passing through a serial-to-parallel $(\mathrm{S} / \mathrm{P})$ converter, each of the modulated complex value symbols is carried by a channel-based orthogonal transform basis. The mapping method is basically done by a direct multiplication operation between each data symbol and the orthogonal basis vector. These orthogonal basis vectors are extracted from the SVD decomposition of the Toeplitz matrix of the channel impulse response $H b_{i j}$ between the $i$ th receiving antenna and the $j$ th transmitting antenna, where $i=1,2$ and $j=1,2$. This can be represented by

$$
H b_{i j}=\left[\begin{array}{ccccc}
h_{i j}[0] & 0 & \ldots & \ldots & 0 \\
\vdots & h_{i j}[0] & 0 & \ddots & \vdots \\
\vdots & \vdots & h_{i j}[0] & \ddots & \vdots \\
h_{i j}[L-1] & \vdots & \vdots & \ddots & 0 \\
0 & h_{i j}[L-1] & \vdots & \ddots & h_{i j}[0] \\
\vdots & 0 & h_{i j}[L-1] & \ddots & \vdots \\
\vdots & \vdots & 0 & \ddots & \vdots \\
0 & 0 & \ldots & \ldots & h_{i j}[L-1]
\end{array}\right]
$$

then,

$$
H b_{j}=\sum_{i=1}^{2} H b_{i j}
$$

where $j=1,2$ and $H b_{j} \in \mathrm{C}^{\left[\left(\frac{\mathrm{N}}{2}+\mathrm{L}-1\right) \times \frac{\mathrm{N}}{2}\right]}$.

The receiver and the transmitter can extract the basic functions by decomposing the Toeplitz matrices using the SVD. Therefore, the Toeplitz matrices can be expressed in terms of the three new matrices as

$$
H b_{j}=U_{n} E_{n} V_{n}^{H}
$$

where $n=1,2$ and $U_{n}$ and $V_{n}^{H}$ are orthogonal matrices that are $\in C^{\left[\left(\frac{N}{2}+\mathrm{L}\right) \times \frac{\mathrm{N}}{2}\right]}$ and $\in \mathrm{C}^{\left[\frac{\mathrm{N}}{2} \times \frac{\mathrm{N}}{2}\right]}$, respectively, and $E_{n}$ is a diagonal matrix with real entires $\in \mathrm{C}^{\left[\frac{\mathrm{N}}{2} \times \frac{\mathrm{N}}{2}\right]}$.

The transformation matrix $V_{n}$ is given by

$$
V_{n}=v_{n}(0) v_{n}(1) \ldots v_{n}\left(\frac{\mathrm{N}}{2}-1\right)
$$

where $v(i)$ is the orthogonal basis vector of length $\left(\frac{\mathrm{N}}{2}-1\right)$. 
The transmitter maps each symbol to its corresponding basis functions by simple multiplication. This leads to two blocks of samples $X_{1}$ and $X_{2}$ referred to two OTDM symbols. This process can mathematically be expressed as

$$
X_{n}=\sum_{i=0}^{\mathrm{N}-1} s_{n}[i] v_{n}[i]=V_{n} S_{n} \ldots \ldots \in \in \mathrm{C}^{\left[\frac{\mathrm{N}}{2} \times 1\right]}
$$

where $n=1,2$ and $V_{n}$ is the Hermitian of $V_{n}^{H}$.

The transmitted data blocks $X_{n}$ of length $\frac{\mathrm{N}}{2}$ can be represented by simple multiplication of Toeplitz matrices $H b_{j} \in \mathrm{C}^{\left[\left(\frac{\mathrm{N}}{2}+\mathrm{L}-1\right) \times \frac{\mathrm{N}}{2}\right]}$ with the transmitted data blocks. The transmitted data blocks pass through a parallel-to-serial $(\mathrm{P} / \mathrm{S})$ converter before adding the guard period (L).

Then, Alamouti space-time coding is applied to $X_{1}$ and $X_{2}$ in the frequency domain to generate the following code word matrix:

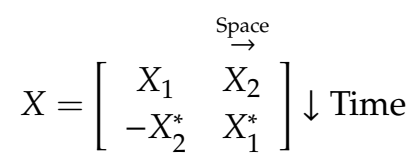

So, the received OTDM signals $R_{i j}$ can be represented as

$$
\begin{gathered}
R_{11}=\left(H_{11} X_{1}\right)+\left(H_{12} X_{2}\right)+z_{1}, \\
R_{21}=\left(H_{21} X_{1}\right)+\left(H_{22} X_{2}\right)+z_{2}, \\
R_{12}=\left(-H_{11} X_{2}^{*}\right)+\left(H_{12} X_{1}^{*}\right)+z_{3}, \text { and } \\
R_{22}=\left(-H_{21} X_{2}^{*}\right)+\left(H_{22} X_{1}^{*}\right)+z_{4},
\end{gathered}
$$

where $i$ is the number of receive antennas and $j$ is the number of transmit antennas, and $z_{1}, z_{2}, z_{3}$, and $z_{4}$ are the additive white Gaussian noise (AWGN) with zero mean and variance $\sigma^{2} . H_{i j}$ are the channel response between the $i$ th receiving antennas and $j$ th transmitting antennas. Then, these signals are exposed to the receiver sequence.

\subsection{STC OTDM Receiver}

At the receiver side, the received signals pass through STC decoder as shown in Figure 2.

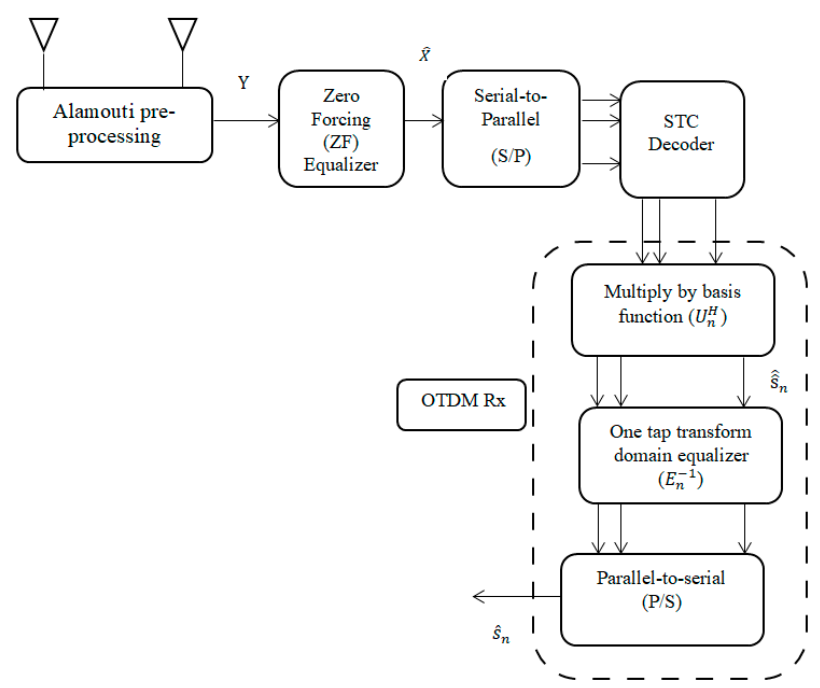

Figure 2. STC OTDM receiver technique. 
The received symbol matrix in the MIMO system can be represented by [9]

$$
\underbrace{\left[\begin{array}{l}
Y_{11} \\
Y_{21} \\
Y_{12}^{*} \\
Y_{22}^{*}
\end{array}\right]}_{Y}=\underbrace{\left[\begin{array}{cc}
\alpha^{*} H_{11} & \alpha^{*} H_{12} \\
\beta^{*} H_{21} & \beta^{*} H_{22} \\
\alpha H_{12}^{*} & -\alpha H_{11}^{*} \\
\beta H_{22}^{*} & -\beta H_{21}^{*}
\end{array}\right]}_{\text {Heq }}\left[\begin{array}{c}
X_{1} \\
X_{1}
\end{array}\right]+\underbrace{\left[\begin{array}{c}
z_{1} \\
z_{2} \\
z_{3} \\
z_{3}
\end{array}\right]}_{Z}
$$

where $\alpha=H_{11}+H_{12}$ and $\beta=H_{21}+H_{22}$.

The ZF equalizer is used to recover the data symbols by considering $\dot{H}_{e q} Y$, where $\dot{H}_{e q}$ is the pseudo-inverse of $H_{e q}$ which can be represented as

$$
\dot{H}_{e q}=\frac{1}{\Lambda} H_{e q}^{H}
$$

where $\Lambda$ is the diversity gain represented as [7]

$$
\Lambda=|\alpha|^{2}\left(\sum_{m=1}^{2}\left|H_{1 m}\right|^{2}\right)+|\beta|^{2}\left(\sum_{m=1}^{2}\left|H_{2 m}\right|^{2}\right)
$$

So, the estimated data symbols can be represented by

$$
\begin{gathered}
\hat{X}=\left[\begin{array}{c}
\hat{X}_{1} \\
\hat{X}_{2}
\end{array}\right]=\left[\begin{array}{cccc}
\alpha H_{11}^{*} & \beta H_{21}^{*} & \alpha^{*} H_{12} & \beta^{*} H_{22} \\
\alpha H_{12}^{*} & \beta H_{22}^{*} & -\alpha^{*} H_{11} & -\beta^{*} H_{21}
\end{array}\right]\left[\begin{array}{c}
Y_{11} \\
Y_{21} \\
Y_{12}^{*} \\
Y_{22}^{*}
\end{array}\right] \text { and } \\
\hat{X}_{n}=X_{n} \Lambda+Z .
\end{gathered}
$$

After that, $\hat{X}$ is passes through the S/P converter followed by the STC decoder. The receiver applies the SVD on the Toeplitz matrix of its channel response. The receiver uses the Hermitian $U_{n}$ as inverse basis functions to extract the data symbols from the received symbols without interference where $n=1,2$. This can be enforced as

$$
\hat{\mathrm{s}}_{n}=\sum_{i=0}^{\mathrm{N}+\mathrm{L}-1} \hat{\hat{x}}_{n}[i] u_{n}^{*}[\mathrm{i}] \ldots \ldots \in \mathrm{C}^{\left[\frac{\mathrm{N}}{2}\right]+\mathrm{L} \times 1} .
$$

Then, a one tap ZF equalization process for $\hat{\hat{s}}_{n}$ is performed by the receiver using $E_{n}$ to get the equalized data symbol block $\hat{s}_{n}$. This process can mathematically be expressed as

$$
\begin{gathered}
\hat{s}_{n}=E_{n}^{-1} \hat{\hat{s}}_{n}=E_{n}^{-1} U_{n}^{\mathrm{H}} \hat{X}_{n} \\
=E_{n}^{-1} U_{n}^{\mathrm{H}}\left(X_{n} \Lambda+Z\right) \\
=E_{n}^{-1} U_{n}^{\mathrm{H}}\left(H b_{j} V_{n} S_{n} \Lambda+Z\right) \\
=E_{n}^{-1} U_{n}^{\mathrm{H}}\left(U_{n} E_{n} V_{n}^{\mathrm{H}} V_{n} S_{n} \Lambda+Z\right) \\
=S_{n} \Lambda+E_{n}^{-1} U_{n}^{\mathrm{H}} Z .
\end{gathered}
$$

This is clearly shown in the system receiver architecture in Figure 2.

\subsection{BER Analysis}

Finally, we need to calculate the power of the signal and the variance of AWGN with zero mean to be able to calculate the BER of the proposed system. So, the power of the signals can be derived as

$$
P_{S}=\sum_{n=1}^{2} E\left[\left|S_{n} \Lambda\right|^{2}\right]
$$

and the noise variance yields to

$$
\sigma^{2}=\sum_{n=1}^{2} E\left[\left|E_{n}^{-1} U_{n}^{H} Z\right|^{2}\right] .
$$


Therefore, the total BER can be represented as [10]

$$
\operatorname{BER}=2 Q\left(\sqrt{\frac{P_{s}}{\sigma^{2}}}\right)\left(1-\frac{1}{2} Q\left(\sqrt{\frac{P_{s}}{\sigma^{2}}}\right)\right)
$$

where $Q($.$) is the standard Q-function.$

Finally, from Equation (22), we proved that the proposed system enhances the performance of the BER.

\section{Simulation Results}

In this section, the proposed STC OTDM technique is evaluated and compared to conventional OFDM [5] and recent multicarrier techniques like STC OFDM [7] and OTDM [14]. Set of experiments are conducted in terms of modulation techniques, number of subcarriers and channel taps. These experiments are discussed in the following subsections. The simulation results are displayed and discussed. An STC OTDM system is considered with $\mathrm{N}=16,64$, and 128 . The modulation schemes QPSK, 64-QAM, and 256-QAM are investigated with a number of channel taps equal to $\mathrm{L}=9,12$ and 15 . For the sake of comparison, a standard OFDM system, STC OFDM and standard OTDM are also considered with $\mathrm{N}=16,64,128$ sub-carriers. In this section, also, the effect of the modulation scheme on all the techniques is studied. The study includes the effect of increasing the number of subcarriers and the effect of increasing the number of channel taps. All the parameters used in simulations are collected in Table 1.

Table 1. Simulation parameters.

\begin{tabular}{cc}
\hline Type of Channel & Rayleigh Fading Channel \\
\hline Number of channel taps, $\mathbf{L}$ & 6,12 and 15 \\
Type of modulation & QPSK, 64-QAM and 256-QAM \\
Number of subcarrier, $\mathbf{N}$ & 16,64 and 128 \\
\hline
\end{tabular}

\subsection{Effect of Modulation Scheme}

Figure 3 shows the BER of the proposed system, compared to the OFDM, STC OFDM and OTDM using QPSK as a modulation technique with a number of subcarriers $\mathrm{N}=16$. It is clear that the BER of the proposed system outperforms the other techniques. The difference in SNR between the proposed system and OTDM technique equals $6 \mathrm{~dB}$ at BER $=10^{-3}$. If we compare the STC OTDM with STC OFDM, we find that at $\mathrm{BER}=10^{-3}$, the difference in SNR between them approximately equals $0.7 \mathrm{~dB}$ and the SNR between the proposed system and the OFDM technique equals $16.5 \mathrm{~dB}$. Furthermore, the eavesdropper cannot decode the data correctly because the eavesdropper and the transmitter are not in the same channel or both have different basis functions as clearly shown in Figure 3.

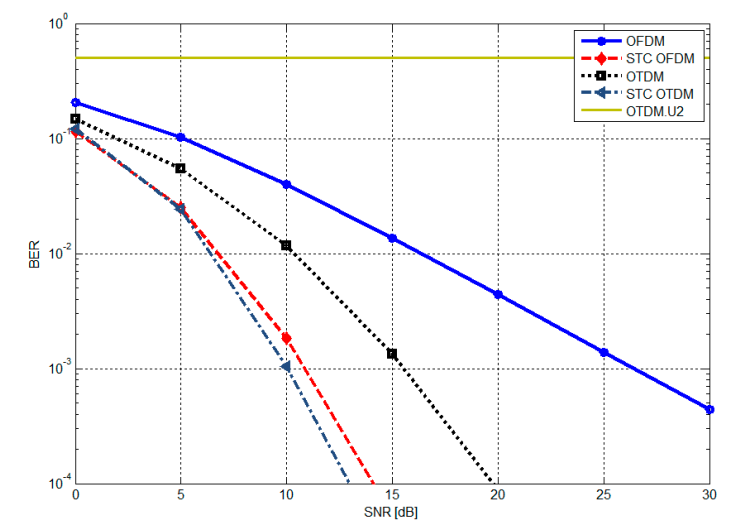

Figure 3. Bit error rate (BER) of the proposed technique (STC OTDM) in comparison with other multicarrier techniques at $\mathrm{N}=16$ and $\mathrm{L}=9$ using QPSK. 
In Figure 4, 64-QAM is used with the same value of N. The effect of increasing the M-ary appears as the BER increases with the modulation order. This is because the constellation becomes closer to each other [16]. This appears at BER $=10^{-3}$, where the difference in SNR between the proposed system and OTDM system is approximately equal to $5 \mathrm{~dB}$, where the SNR of the proposed system is $22.5 \mathrm{~dB}$ and $10 \mathrm{~dB}$ at 64-QAM and QPSK, respectively, at BER $=10^{-3}$. Thus, in 256-QAM, the performance of the proposed system degrades to reach $28 \mathrm{~dB}$ at the same BER as shown in Figure 5.

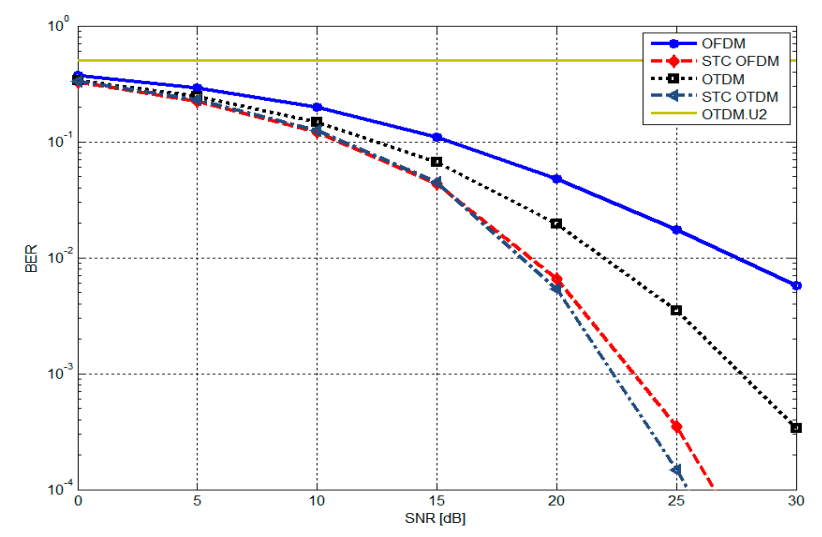

Figure 4. BER of the proposed technique (STC OTDM) in comparison with other multicarrier techniques at $\mathrm{N}=16$ and $\mathrm{L}=9$ using 64-QAM.

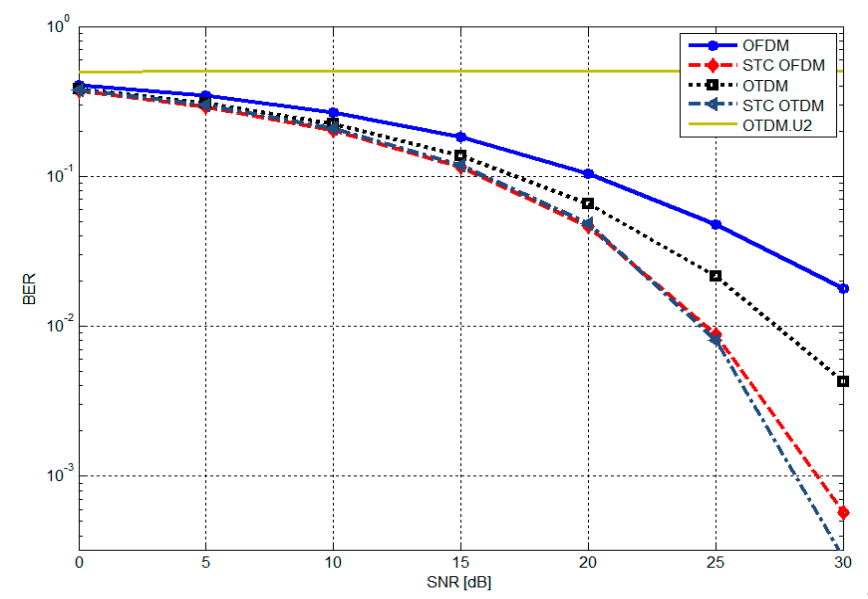

Figure 5. BER of the proposed technique (STC OTDM) in comparison with other multicarrier techniques at $\mathrm{N}=16$ and $\mathrm{L}=9$ using 256-QAM.

\subsection{Effect of Subcarriers}

Figure 6 shows the effect of increasing the number of subcarriers at all modulation techniques. The proposed system gives SNR $=10 \mathrm{~dB}$ at $\mathrm{BER}=10^{-3}$ at QPSK modulation technique when $\mathrm{N}=16$. The performance of STC OTDM when $\mathrm{N}=64$ and 128 is nearly equal. So, this is great evidence to prove that STC OTDM gives high performance with less number of subcarriers at all the modulation techniques as it clear in the figure. The performance decreases at 64-QAM and 256-QAM to $\mathrm{SNR}=22.5 \mathrm{~dB}$ and $28 \mathrm{~dB}$, respectively, at $\mathrm{BER}=10^{-3}$. Thus, the required performance can be obtained with a lower number of subcarriers and then lower complexity. This is in addition to the fact that the proposed technique is based on linear equalization and an SVD algorithm which is widely used in different present wireless communication systems like in channels estimation and beamforming algorithms [15]. The complexity of the SVD computation is of order $\mathrm{O}\left(m n^{2}\right)$, where $\mathrm{m}$ and $\mathrm{n}$ are the matrix dimensions that will apply the SVD on it [17]. Thus, the proposed system is applicable and can practically be deployed. 


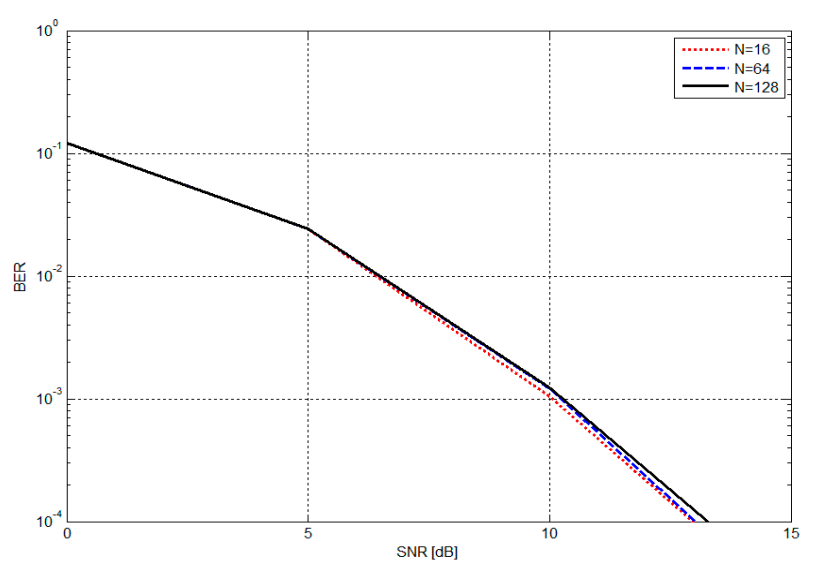

(a)

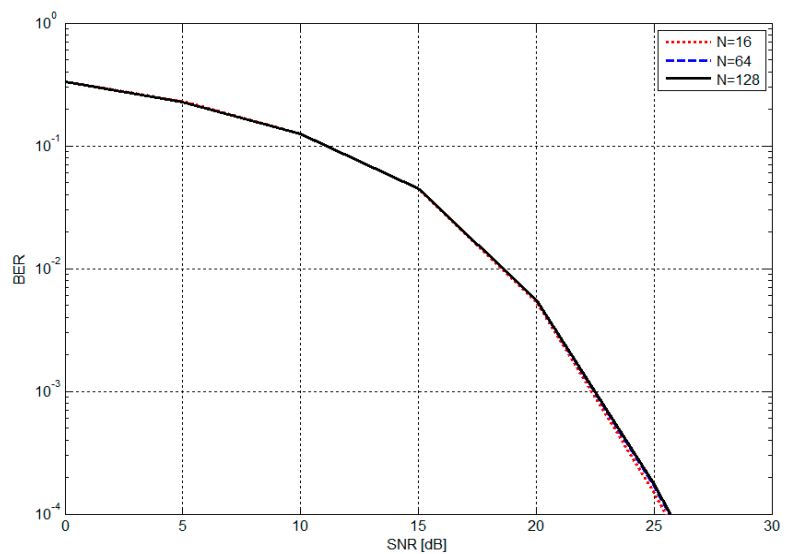

(b)

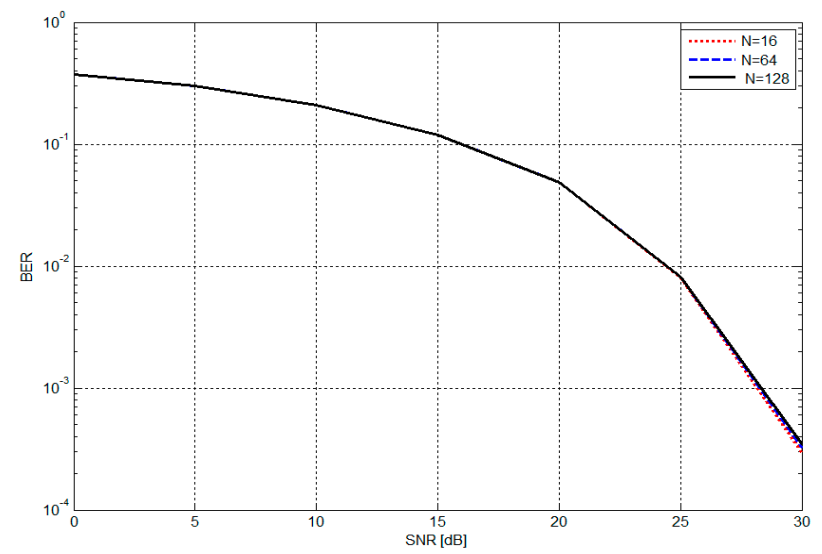

(c)

Figure 6. BER of STC OTDM at different subcarriers and L = 9 for: (a) QPSK; (b) 64-QAM; and (c) 256-QAM.

\subsection{Effect of Channel Taps}

The effect of increasing the taps of the channel on the proposed system appears in Figure 7. Increasing the channel taps from 9 to 12 leads to a little bit increase in the performance of the proposed system at SNR greater than $10 \mathrm{~dB}$. This is in a fair agreement with the work of Hamamreh and Arslan in [14] as the OTDM is designed especially to work better with the frequency selective channels, which is the case in most broadband systems. 


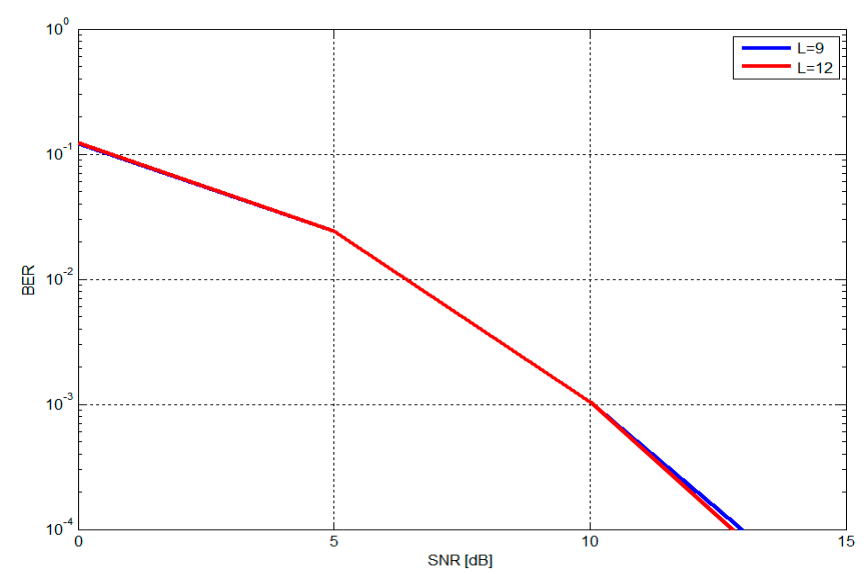

Figure 7. BER of STC OTDM at different channel taps L using QPSK.

The procedure is repeated for all modulation schemes at all values of $\mathrm{N}$ with channel taps of 9, 12, and 15. The obtained results at BER $=10^{-3}$ are summarized in Table 2 .

Table 2. The SNR (dB) simulation results at BER $=10^{-3}$ for different modulation schemes, different subcarriers, and channels models.

\begin{tabular}{|c|c|c|c|c|c|c|c|c|c|}
\hline \multirow[b]{2}{*}{ L } & \multicolumn{9}{|c|}{ SNR (dB) } \\
\hline & \multicolumn{3}{|c|}{9} & \multicolumn{3}{|c|}{12} & \multicolumn{3}{|c|}{15} \\
\hline $\mathbf{N}$ & 16 & 64 & 128 & 16 & 64 & 128 & 16 & 64 & 128 \\
\hline \multicolumn{10}{|c|}{ QPSK } \\
\hline OFDM & 26.5 & 27 & 27 & 26.8 & 27 & 27 & 26.4 & 26.6 & 26.8 \\
\hline STC OFDM & 10.7 & 11 & 11 & 10.7 & 10.9 & 10.9 & 10.9 & 11 & 11 \\
\hline OTDM & 16 & 22 & 24 & 14 & 21 & 23.2 & 13.4 & 20 & 22.4 \\
\hline STC OTDM & 10 & 10.1 & 10.1 & 10 & 10.01 & 10.01 & 10 & 10.01 & 10.01 \\
\hline \multicolumn{10}{|c|}{ 64-QAM } \\
\hline OFDM & 34 & 35 & 35 & 35 & 35.2 & 35.1 & 35.2 & 35.3 & 35.4 \\
\hline STC OFDM & 23 & 23 & 23 & 23.2 & 23.15 & 23.1 & 23.2 & 23 & 23 \\
\hline OTDM & 27.5 & 31 & 31.4 & 26.4 & 32.7 & 32.7 & 25.4 & 31.5 & 33 \\
\hline STC OTDM & 22.3 & 22.4 & 22.4 & 22.2 & 22.4 & 22.4 & 22 & 22.3 & 22.4 \\
\hline \multicolumn{10}{|c|}{ 256-QAM } \\
\hline OFDM & 37.7 & 38.5 & 39 & 38.5 & 39 & 39.3 & 39.8 & 39.9 & 38.6 \\
\hline STC OFDM & 28.3 & 28.5 & 28.7 & 28.2 & 28.4 & 28.29 & 29 & 28.89 & 28.96 \\
\hline OTDM & 32.5 & 37 & 37.4 & 31 & 36.8 & 37 & 32 & 36 & 37 \\
\hline STC OTDM & 28.2 & 28.3 & 28.32 & 28 & 28.2 & 28.28 & 28.1 & 28.12 & 28.2 \\
\hline
\end{tabular}

\section{Conclusions}

An STC OTDM architecture is proposed for enhancing the performance of the BER and increasing the diversity order. We considered a system with $\mathrm{N}=16,64$, and 128 . The modulation schemes QPSK, 64-QAM, and 256-QAM were investigated with a number of channel taps equaling 9, 12, and 15 . For the sake of comparison, we also considered a standard OFDM system, STC OFDM, and standard OTDM with the same active sub-carriers, a cyclic prefix (CP) of length $L$ for the OFDM and STC OFDM techniques, and simple zero-forcing equalization. The proposed system shows its superiority over the OFDM, STC OFDM, and OTDM in enhancing the performance of BER. The simulation results show that the SNR of the proposed system using QPSK modulation technique is equal to $10 \mathrm{~dB}$ at $\mathrm{BER}=10^{-3}$ when 16 subcarriers are used in a 9-tap Rayleigh fading channel. Also, the results show that the BER performance of the proposed technique is nearly constant for a different number of subcarriers. For example, the proposed technique using 64-QAM achieved the same BER performance, $10^{-3}$ at 
$\mathrm{SNR}=22.5 \mathrm{~dB}$, for the different subcarriers 64 and 128. Thus, the required system performance can be obtained with less number of subcarriers and thus less system complexity for the different modulation techniques. When using 64-QAM and 256-QAM the performance of BER is nearly the same and the proposed system achieves high performance at high data rates. Simulations also showed that the STC OTDM improves the security of the wireless communication system, where only the desired user can decode the transmitted data.

The proposed technique is basically composed of a linear equalizer and SVD algorithm which is widely used in different present wireless communication systems. The complexity of the SVD computation is of order $\mathrm{O}\left(m n^{2}\right)$ where $\mathrm{m}$ and $\mathrm{n}$ are the matrix dimensions that will apply the SVD on it. Thus, the proposed system is applicable and can be practically deployed.

Author Contributions: Conceptualization, A.Z. and W.K.B.; Methodology, R.M.A., A.Z. and W.K.B.; Software, R.M.A., A.Z. and W.K.B.; Validation, A.Z., W.K.B. and M.H.A.; Formal Analysis, A.Z. and W.K.B.; Investigation, R.M.A.; Resources, R.M.A., A.Z. and W.K.B.; Data Curation, R.M.A.; Writing-Original Draft Preparation, R.M.A.; Writing-Review \& Editing, A.Z., W.K.B. and M.H.A.; Visualization, R.M.A., A.Z. and W.K.B.; Supervision, A.Z., W.K.B. and M.H.A.; Project Administration, R.M.A., A.Z. and W.K.B.

Funding: This research has no external funding.

Conflicts of Interest: The authors declare no conflict of interest.

\section{References}

1. Vij, S.; Jain, A. 5G: Evolution of a secure mobile technology. In Proceedings of the 2016 3rd International Conference on Computing for Sustainable Global Development (INDIACom), New Delhi, India, 16-18 March 2016; pp. 2192-2196.

2. Yang, N.; Wang, L.; Geraci, G.; Elkashlan, M.; Yuan, J.; Renzo, M.D. Safeguarding 5G wireless communication networks using physical layer security. IEEE Commun. Mag. 2015, 53, 20-27. [CrossRef]

3. Gerzaguet, R.; Medjahdi, Y.; Demmer, D.; Zayani, R.; Dor, J.B.; Shaiek, H.; Roviras, D. Comparison of promising candidate waveforms for 5G: WLOA-OFDM versus BF-OFDM. In Proceedings of the 2017 International Symposium on Wireless Communication Systems (ISWCS), Bologna, Italy, 28-31 August 2017; pp. 355-359.

4. Boroujeny, B.F; Moradi, H. OFDM inspired waveforms for 5G. IEEE Commun. Surv. Tutor. 2016, 18, 2474-2492. [CrossRef]

5. Goldsmith, A. Wireless Communications; Cambridge University Press: Cambridge, UK, 2005.

6. Li, Y.; Stüber, G.L. Orthogonal Frequency Division Multiplexing for Wireless Communications; Springer: New York, NY, USA, 2006.

7. Bharti, P.K.; Rawat, P. Alamouti-STBC based performance estimation of multi Tx and Rx antenna over MIMO-OFDM. In Proceedings of the 2018 2nd International Conference on Trends in Electronics and Informatics (ICOEI), Tirunelveli, India, 11-12 May 2018; pp. 1277-1281.

8. Alamouti, S. A simple transmit diversity technique for wireless communications. IEEE J. Sel. Areas Commun. 1998, 16, 1451-1458. [CrossRef]

9. Fernando, N.; Hong, Y.; Viterbo, E. MIMO self-heterodyne OFDM. IEEE Trans. Veh. Technol. 2015, 65, 1271-1280. [CrossRef]

10. Fernando, N.; Hong, Y.; Viterbo, E. Self-heterodyne OFDM transmission for frequency selective channels. IEEE Trans. Commun. 2013, 61, 1936-1946. [CrossRef]

11. Bariah, L.; Al-Dweik, A.; Muhaidat, S. On the performance of non-orthogonal multiple access systems with imperfect successive interference cancellation. In Proceedings of the 2018 IEEE International Conference on Communications Workshops (ICC Workshops), Kansas City, MO, USA, 20-24 May 2018; pp. 1-6.

12. Ghaffari, A.; Leonardon, M.; Cassagne, A.; Leroux, C.; Savaria, Y. Toward high-performance implementation of 5G SCMA algorithms. IEEE J. Mag. 2019, 7, 10402-10414. [CrossRef]

13. Hadani, R.; Rakib, S.; Molisch, A.F.; Ibars, C.; Monk, A.; Tsatsanis, M.; Delfeld, J.; Goldsmith, A.; Calderbank, R. Orthogonal time frequency space (OTFS) modulation for millimeter-wave communications systems. In Proceedings of the 2017 IEEE MTT-S International Microwave Symposium (IMS), Honololu, HI, USA, 4-9 June 2017; pp. 681-683. 
14. Hamamreh, J.M.; Arslan, H. Secure orthogonal transform division multiplexing (OTDM) waveform for 5G and beyond. IEEE Commun. Lett. 2017, 21, 1191-1194. [CrossRef]

15. Alluhaibi, O.; Nair, M.; Hazzaa, A.; Mihbarey, A.; Wang, J. 3D beamforming for 5G millimeter wave systems using singular value decomposition and particle swarm optimization approaches. In Proceedings of the 2018 International Conference on Information and Communication Technology Convergence (ICTC), Jeju Island, Korea, 17-19 October 2018; pp. 15-19.

16. Sadinov, S.M. Simulation study of M-ARY QAM modulation techniques using Matlab/Simulink. In Proceedings of the 2017 40th International Convention on Information and Communication Technology, Electronics and Microelectronics (MIPRO), Opatija, Croatia, 22-26 May 2017; pp. 547-554.

17. Golub, G.H.; Van Loan, C.F. Matrix Computations; The Johns Hopkins University Press: Baltimore, MD, USA, 2013.

(C) 2019 by the authors. Licensee MDPI, Basel, Switzerland. This article is an open access article distributed under the terms and conditions of the Creative Commons Attribution (CC BY) license (http://creativecommons.org/licenses/by/4.0/). 